\title{
EchoGéo
}

35 | 2016

Les découpages territoriaux en Europe

\section{La réforme territoriale en France : parlementaires et géographes face à "l'art de la découpe » gouvernementale}

Alexis Sierra et Élisabeth Bonnet-Pineau

\section{CpenEdition}

Journals

Édition électronique

URL : https://journals.openedition.org/echogeo/14481

DOI : 10.4000/echogeo.14481

ISSN : 1963-1197

Éditeur

Pôle de recherche pour l'organisation et la diffusion de l'information géographique (CNRS UMR 8586)

Référence électronique

Alexis Sierra et Élisabeth Bonnet-Pineau, « La réforme territoriale en France : parlementaires et géographes face à « l'art de la découpe » gouvernementale », EchoGéo [En ligne], 35 | 2016, mis en ligne le 19 avril 2016, consulté le 11 août 2021. URL : http://journals.openedition.org/echogeo/14481 ; DOI : https://doi.org/10.4000/echogeo.14481

Ce document a été généré automatiquement le 11 août 2021.

EchoGéo est mis à disposition selon les termes de la licence Creative Commons Attribution - Pas d'Utilisation Commerciale - Pas de Modification 4.0 International (CC BY-NC-ND) 


\title{
La réforme territoriale en France : parlementaires et géographes face à "l'art de la découpe » gouvernementale
}

\author{
Alexis Sierra et Élisabeth Bonnet-Pineau
}

1 La loi du 16 janvier 2015 relative à la délimitation des régions modifie la carte des régions françaises. À compter du $1^{\text {er }}$ janvier 2016, l'article $1^{\text {er }}$ de la loi du 16 janvier 2015 substitue 13 Régions aux 22 Régions métropolitaines existantes.

2 L'objectif est de doter la France de régions de taille équivalente à celle des autres régions européennes pour gagner en efficience et ... moins solliciter le contribuable.

3 Avec cet objectif, le gouvernement, puis le parlement, se sont ainsi essayés à un redécoupage territorial, un "art de la découpe » qui intéresse particulièrement le géographe. En effet, le géographe, par et pour ses analyses crée des catégories spatiales et territoriales, et en conséquence peut lui-même être consulté, tel Vidal de la Blache au sortir de la Grande Guerre, pour établir une régionalisation du pays. Néanmoins, plusieurs géographes rappellent que diviser le territoire pour l'étudier ou le diviser pour le gérer ne relève pas des mêmes objectifs, et que la finalité du géographe est davantage dans l'analyse des raisons du découpage régional que dans la proposition aux politiques de ce découpage (Brunet, 1997 ; Giblin, 1986).

4 Le politique, parce qu'il exerce sur un territoire, doit circonscrire l'aire de son pouvoir, et en organiser sa représentation et sa gestion. La loi actuelle n'est en ce sens qu'une étape supplémentaire dans le travail de découpe que gouvernements et parlements ont opéré dans l'histoire du pays. Citons la création des départements en 1790, les régions économiques de programme en $1955^{1}$ qui sont devenues des collectivités territoriales de plein exercice en 1982, à quoi il faut ajouter les découpages en circonscriptions électorales à différentes échelles, les circonscriptions législatives étant celles qui ont fait couler le plus d'encre. Dans ce dernier cas, le découpage s'appuie en tout premier lieu sur les rapports de forces identifiés, même s'il intègre certains critères issus de 
l'analyse du territoire, telle la composante démographique, et même si le savoir géographique est alors utilisé pour légitimer la découpe (Erhard, 2011). "Les découpeurs d'espace aiment la géographie. C'est un inépuisable fournisseur de légitimité » écrivait Roger Brunet qui voyait dans l'appel aux géographes, le besoin de «naturaliser " un découpage éminemment politique en faisant appel à des données structurelles et apparemment pérennes (Brunet, 1997). Rappelons également que l'utilisation de cette notion de découpe n'était pas exempte pour Roger Brunet d'ironie distanciée et que dans ce même article de référence il considère, ironie de l'histoire que «nul ne croit plus que l'on changera en France, les limites départementales à terme prévisible, ni même les limites régionales ". Moins de vingt ans après, la première affirmation se confirme toujours et la seconde n'est infirmée que partiellement.

5 En effet, les départements existent toujours en l'état et bien qu'un débat sur leur suppression persiste, ils ont été confirmés par la dernière réforme, leur existence étant au demeurant constitutionnelle. Quant aux régions, plus que d'un redécoupage réel, cette loi a procédé à des fusions de régions existantes, sans recomposer les anciennes, sans attribuer les différents départements d'une région à de nouvelles régions distinctes, comme le demandait par exemple plusieurs acteurs de la Loire-Atlantique. La marge de manœuvre des parlementaires était très réduite. Dans l'interview qui suit, Jean-Jacques Hyest montre que même sans adhérer aux arguments justifiant la réduction du nombre de régions, les parlementaires se sont inscrits dans cet objectif pour tenter de peser sur le choix des fusions bien qu'au final, il n'a pas été possible pour eux de modifier substantiellement le projet de loi.

6 Suite à la publication par le gouvernement de la nouvelle carte des régions, plusieurs experts, notamment des chercheurs en sciences sociales, sont intervenus dans les media et ont publié des tribunes pour donner un avis, soit pour analyser la partition proposée, soit pour suggérer un maillage à leurs yeux plus rationnel ou du moins, proposer des critères sur lesquels celui-ci devrait se fonder. Une multiplicité de découpages sont alors possibles en fonction des critères retenus, ce que certains journaux ont montré, non sans rappeler les diverses tentatives de découpage régional existant depuis l'Entre-deux-guerres ni les multiples circonscriptions régionales utilisées par les administrations et les entreprises (Académies de l'Éducation nationale, circonscriptions téléphoniques, régions militaires, etc...) (Marconis, 2006).

7 Ces experts intervenus sur la place publique ne sont pas tous des géographes. Bien que la question posée soit éminemment territoriale, nous voyons à cette occasion d'autres spécialistes la traiter et rappeler à notre communauté scientifique que si le territoire et la région sont des objets privilégiés de la géographie celle-ci n'en a pas le monopole.

Quand le gouvernement dépose son projet au Sénat, celui-ci décide de recueillir l'avis d'une partie de ces experts, en mettant en place, dans un temps extrêmement contraint et limité, une commission spéciale, présidée par Jean-Jacques Hyest, sénateur LR de la Seine-et-Marne. Cette commission a opéré une série d'auditions. Ont été ainsi entendus: un démographe (Hervé Le Bras), un sociologue (Patrick Le Lidec), un politiste (Romain Pasquier) et 5 géographes: Daniel Béhar, Professeur à l'Institut d'urbanisme de Paris, Gérard-François Dumont, Professeur à l'Université de Paris IV, Jacques Lévy, professeur à l'École Polytechnique de Lausanne, Philippe Subra, Professeur à l'Université de Paris 8 et Martin Vanier, Professeur à l'Université Joseph Fournier de Grenoble. Certains étaient déjà connus des sénateurs ou enregistrés par les services du Sénat, d'autres s'étaient faits connaître par leur intervention dans les 
media. Réunis en table ronde, ils se sont exprimés successivement, leurs propos étant consignés dans le rapport de la commission spéciale accessible au public ${ }^{2}$ et sur lequel nous nous appuyons. Notre objectif est ici de réfléchir au sens de ces consultations publiques et de voir comment les géographes peuvent éclairer le débat politique, quelles approches de la géographie ils mobilisent dans ce débat, tout en mettant en perspective l'interview de Jean-Jacques Hyest.

\section{Le point de vue des géographes consultés}

9 La position de ces experts est bien d'éclairer la décision publique par leur connaissance $\mathrm{du}$ territoire. Cependant ce sont de véritables thèses qui sont proposées, dans un engagement à la fois scientifique et citoyen, oscillant entre la géographie active d'un Pierre George et la géographie appliquée d'un Michel Philiponneau. La plupart d'entre eux regrettent ainsi l'absence de grand débat national ou du moins l'absence de temps pour une large consultation, Philippe Subra rappelle ainsi que la création des départements avait eu comme préalable une consultation des différentes parties du territoire durant 6 mois. Ils n'hésitent pas à critiquer certaines fusions ou à réclamer le démembrement de certaines régions existantes (La Picardie par exemple). La prise de position peut devenir véhémente et montrer l'instrumentalisation de ce découpage comme un moyen pour détourner l'attention des problèmes de fond que pose l'organisation territoriale : « cette carte est un leurre » affirme ainsi Martin Vanier.

10 L'un des résultats de la consultation a été de battre en brèche certaines idées fausses nous dit Jean Jacques Hyest dans son interview. On retrouve cette idée dans le compterendu des auditions. Jacques Lévy, Martin Vanier, Philippe Subra et plus encore GérardFrançois Dumont remettent en cause le critère de la taille, chiffres à l'appui : les comparaisons avec d'autres pays européens montre que les 21 régions françaises n'étaient pas hors norme, que Midi-Pyrénées et Rhône-Alpes étaient déjà plus grandes que certains États européens et que l'écart entre la plus petite et la plus grande était moindre en France qu'en Allemagne, en Espagne ou en Italie. Daniel Béhar adopte toutefois une position plus nuancée, plus opérationnelle, celle de l'aménageur qui voit dans la constitution de grandes régions, l'émergence « d'instances de fabrication des politiques publiques ».

11 Contrairement à la position pro-départementale du sénateur Hyest, la place de l'urbain chez certains des géographes consultés est révélatrice d'une forme de penser le territoire contemporain. Jacques Lévy ou Daniel Béhar adoptent ainsi un point de vue qui part de l'urbain, insistant sur la priorité à donner à la métropolisation ou à la nécessaire articulation entre région et métropole. Daniel Béhar et plus encore Martin Vanier, qui adopte une attitude résolument "post-territoriale », en appellent à une réflexion sur l'espace réticulaire, dans laquelle l'action régionale s'articule en réseau à partir des métropoles. Une autre approche symptomatique de la géographie contemporaine, et qui rejoint certains propos du sénateur, est la réflexion sur la dimension identitaire: Philippe Subra ou Jacques Lévy insistent sur la nécessaire prise en compte des identités dans la construction des régions, le second montrant que « la région est un espace biographique, c'est-à-dire qu'elle offre à chacun un éventail d'opportunités pour réaliser des projets (...) un point d'équilibre entre des ressources objectives, en gros les métropoles, et des ressources subjectives, les identités ». On notera également chez ces deux derniers auteurs une approche historique qui n'est pas 
sans rappeler la démarche géopolitique, avec pour Jacques Lévy une claire prise de position girondine, décentralisatrice et métropolitaine, montrant notamment que les choix opérés sous la Troisième République «en congelant le projet révolutionnaire dynamique et fédéral » puis en 1982 « en renforçant des niveaux qui n'avaient pas lieu d'être ", ont rendu la réforme territoriale plus difficile.

Aucun d'eux ne fait cependant d'analyse proprement géopolitique du découpage proposé. Non reconnu par le gouvernement mais largement commenté dans la presse, le rôle de certains leader régionaux proche du gouvernement a été pourtant déterminant et nous a été confirmé par Jean-Jacques Hyest. La consultation révèle le caractère incomplet ou inachevé de la réforme. Le redécoupage territorial n'est qu'une composante d'une réforme régionale dans laquelle la clarification des compétences entre les différents échelons politico-administratifs était attendue depuis des années. Ce découpage n'a finalement consisté qu'en un ajustement.

La consultation des géographes n'a pas permis de changer le cours de la réforme mais elle a légitimé le discours des opposants à la réforme qui étaient majoritaires au Sénat. Nous retrouvons cette ambivalence analysée par Thomas Ehrhart (2011) dans le cas du découpage électoral, entre d'une part, le nécessaire appel à un savoir expert, et de l'autre son instrumentalisation à des fins de légitimation de l'action publique. En outre, l'entretien réalisé avec Jean-Jacques Hyest montre que la circulation des arguments des géographes vers les parlementaires fait de ces commissions un lieu de rencontre entre deux pratiques et deux vision du territoire, et une instance de légitimation de la parole publique y compris pour celle des scientifiques, qui voient ainsi leurs compétences reconnues.

L'interview qui suit a été réalisée en deux temps, avant et après le changement de fonction de Jean-Jacques Hyest, nommé par Gérard Larcher membre du conseil constitutionnel en novembre 2015. Nous le remercions de son accueil et d'avoir consacré du temps à ces entretiens qui se sont déroulés en toute liberté, dans ses bureaux du Sénat et de la rue Montpensier. Cette interview, validée par ses soins est une proposition adressée à toutes celles et ceux qui souhaitent réfléchir plus avant sur la position des géographes comme consultants et experts du politique, et sur leur apport dans les débats législatifs.

\section{BIBLIOGRAPHIE}

Brunet R., 1997. Territoires : art de la découpe. Revue de Géographie de Lyon. Vol. 72-3/97.

Ehrhard T., 2011. Dualité théorique et pratique : le découpage électoral au prisme révélateur de la mobilisation des savants et des savoirs. Actes du Congrès AFSP Strasbourg 2011.

Giblin B., 1986. Introduction. La région, un territoire politique. In Yves Lacoste, Géopolitiques des régions françaises, tome I, Paris, La France septentrionale..., 1986, p. 34.

Marconis R., 2006. France : recompositions territoriales. La Documentation photographique. 
NOTES

1. Décret du 30 juin 1955.

2. Projet de loi relatif à la délimitation des régions, aux élections régionales et départementales et modifiant le calendrier électoral (http://www.senat.fr/rap/l13-658/l13-658.html) 